\title{
SERIES EXPANSIONS OF RAYS IN ISOTROPIC, NON-HOMOGENEOUS MEDIA*
}

\author{
By SAUL GORN (Aberdeen Proring Ground)
}

The interest in air-to-air radio propagation and its possible use for high-accuracy distance measuring make it useful to have available recursive formulae for the coefficients of the power series expansions of rays. The following note has been extracted from a government report (see Gorn, [6]) with this purpose in mind.

It will be convenient to use a combination of vector and matrix notations. Thus a ray will be given by its position vector: $\mathbf{x}=\left(x_{1}(t), x_{2}(t), x_{3}(t)\right)$. This is a row vector and a $1 \times 3$ matrix; the corresponding column vector will be indicated by $\mathbf{x}^{T}$. Thus the dot product of two vectors is simply $\mathbf{y} \cdot \mathbf{z}=\mathrm{yz}^{T}$, while $\mathrm{y}^{T} \mathbf{z}$ is a $3 \times 3$ matrix. The unit $3 \times 3$ matrix, with ones down the main diagonal, and zeroes elsewhere, will be represented by $I$. $\mathbf{y}^{2}$ will mean $\mathbf{y} \cdot \mathbf{y}=\mathrm{yy}^{T}$.

It will also be convenient to use the normalized time parameter

$$
u=c t \text {. }
$$

Here $c$ is the speed of light in a vacuum. Differentiation will be with respect to $u$, so that, for example, $\mathbf{x}^{\prime}$ will mean the column vector $(\mathrm{a} 3 \times 1$ matrix) whose components are $d x_{i} / d u, i=1,2,3$. Since the index of refraction, $n\left(x_{1}, x_{2}, x_{3}\right)$, is defined as $c$ divided by the speed of light in the medium, it follows that along a ray $\mathbf{x}$ we have

$$
\mathbf{x}^{\prime 2}=n^{-2} \text {. }
$$

It is at this point that the isotropic property of the medium is used.

Of the various standard forms for the differential equations of rays, which can be found in Herzberger [1], Synge [3], and Luneberg [4], we will therefore use

$$
\left\{n\left(\mathbf{x}^{\prime 2}\right)^{-1 / 2} \mathbf{x}^{\prime}\right\}^{\prime}=\left(\mathbf{x}^{\prime 2}\right)^{1 / 2} \nabla n,
$$

where $\nabla n$ means, of course, the gradient of $n$ :

$$
\nabla n=\left(\frac{\partial n}{\partial x_{1}}, \frac{\partial n}{\partial x_{2}}, \frac{\partial n}{\partial x_{3}}\right) .
$$

Equation (3) is usually simplified by immediate use of equation (2), but new forms of these equations which have direct geometric interpretations can be most readily derived by postponing such use.

First note that from the chain rule for differentiation

$$
n^{\prime}=\mathbf{x}^{\prime} \cdot \nabla n \text {. }
$$

Also, differentiating (2) yields

$$
\mathbf{x}^{\prime} \cdot \mathbf{x}^{\prime \prime}=-n^{-3} n^{\prime}=-n^{-3}\left(\mathbf{x}^{\prime} \cdot \nabla n\right) .
$$

If, then, we perform directly the differentiation of the fraction indicated in the left hand side of (3), and then clear (3) of fractions, a use of (2) and (4), a transposition and a multiplication by $n$ yield

$$
n^{-3} \nabla n-n^{-1}\left(\mathbf{x}^{\prime} \cdot \nabla n\right) \mathbf{x}^{\prime}=\mathbf{x}^{\prime \prime}-n^{2}\left(\mathbf{x}^{\prime} \cdot \mathbf{x}^{\prime \prime}\right) \mathbf{x}^{\prime} .
$$

${ }^{*}$ Received January 28, 1953. 
If in (6) we transpose all matrices, we get

$$
\left\{I-n^{2} \mathbf{x}^{\prime T} \mathbf{x}^{\prime}\right\} \mathbf{x}^{\prime \prime T}=\left\{I-n^{2} \mathbf{x}^{\prime T} \mathbf{x}^{\prime}\right\}\left(n^{-3} \nabla n\right)^{T} .
$$

On the other hand we could use (5) either on the left or on the right of (6), transpose a term and then transpose all matrices to obtain the following two forms:

$$
\begin{aligned}
\mathbf{x}^{\prime \prime T} & =\left\{I-2 n^{2} \mathbf{x}^{\prime T} \mathbf{x}^{\prime}\right\}\left(n^{-3} \nabla n\right)^{T}, \\
\left(n^{-3} \nabla n\right)^{T} & =\left\{I-2 n^{2} \mathbf{x}^{\prime T} \mathbf{x}^{\prime}\right\} \mathbf{x}^{\prime \prime} .
\end{aligned}
$$

It is not difficult to show, as is done in Gorn (6), that $I-2 n^{2}{\mathbf{x}^{\prime}}^{T} \mathbf{x}^{\prime}$ is the symmetry operator through the plane perpendicular to $\mathbf{x}^{\prime}$, while $I-n^{2} \mathbf{x}^{\prime T} \mathbf{x}^{\prime}$ is the projection operator on to that plane. Equations (8) therefore mean that $\mathbf{x}^{\prime \prime}$ and $n^{-3} \nabla n$ are symmetric images through the plane perpendicular to $\mathbf{x}^{\prime}$, while equation (7) states that they have the same projection on to that plane. The three component equations of (7) must, consequently, be dependent, since many vectors have the same projection as $n^{-3} \nabla n$; (7) contains incomplete information about $\mathbf{x}^{\prime \prime}$ and needs, say, (2) as an adjunct. Either set of (8), on the other hand, contains complete information.

At this point we can make use of the standard differential geometry of space curves (see e.g. Franklin [5], p. 107 on) with its concepts of curvature $\kappa$, torsion $\tau$, the natural coordinate vectors, namely the unit tangent $\mathbf{T}$, the unit normal $\mathbf{N}$, and the unit binormal B, and finally the Frenet-Serret formulae expressing the derivatives of the natural coordinate vectors as linear combinations of $\mathbf{T}, \mathbf{N}$, and $\mathbf{B}$. Once we find the curvature and torsion along rays we will be able to differentiate any vector field defined along them by using the Frenet-Serret formulae. In particular, repeated differentiation of $\mathbf{x}$ by this means will yield power series expansions of rays. These expansions correspond to the one well-known in differential geometry for space curves.

If $s$ is the arc length measured along a ray, then $s^{\prime}=n^{-1}$. Furthermore, the transpose of the first form of $(8)$ is

$$
\mathbf{x}^{\prime \prime}=-2 n^{-1}\left(\mathbf{x}^{\prime} \cdot \nabla n\right) \mathbf{x}^{\prime}+n^{-3} \nabla n .
$$

From these and the curvature formula, one readily derives the well-known formulae for the curvature of a ray:

$$
\kappa=\left|\mathbf{x}^{\prime} \times \nabla n\right|=-n^{-1}|\nabla n| \sin \psi,
$$

where $\psi$ is the angle between $\mathbf{x}^{\prime}$ and $\nabla n$.

The standard forms for $\mathbf{T}, \mathbf{N}$, and $\mathbf{B}$, with the help of equations (5) and (9) become

$$
\begin{aligned}
& \mathbf{T}=\quad n \mathbf{x}^{\prime} \\
& \mathbf{N}=-n \kappa^{-1}\left(\mathbf{x}^{\prime} \cdot \nabla n\right) \mathbf{x}^{\prime}+(n \kappa)^{-1} \nabla n \\
& \mathbf{B}=\quad \kappa^{-1} \mathbf{x}^{\prime} \times \nabla n \text {. }
\end{aligned}
$$

.To find a formula for the torsion of a ray, one differentiates (9), and uses the rules or simplifying a determinant to obtain the scalar triple product

$$
\left(\mathbf{x}^{\prime} \mathbf{x}^{\prime \prime} \mathbf{x}^{\prime \prime \prime}\right)=n^{-6}\left(\mathbf{x}^{\prime} \nabla n[\nabla n]^{\prime}\right) .
$$

Since

$$
[\nabla n]^{\prime}=\mathbf{x}^{\prime} \nabla_{2} n,
$$


where

$$
\nabla_{2} n=\left(\frac{\partial^{2} n}{\partial x_{i} \partial x_{i}}\right)
$$

a symmetric matrix, the standard torsion formula yields

$$
\tau=\kappa^{-2}\left(\mathbf{x}^{\prime} \nabla n[\nabla n]^{\prime}\right)=\kappa^{-2}\left(\mathbf{x}^{\prime} \nabla n \mathbf{x}^{\prime} \nabla_{2} n\right) .
$$

Alternative forms for $\kappa$ and $\tau$ can be given in terms of the matrix

$$
Y=\left(\begin{array}{ccc}
0 & -\frac{\partial n}{\partial x_{3}} & \frac{\partial n}{\partial x_{2}} \\
\frac{\partial n}{\partial x_{3}} & 0 & -\frac{\partial n}{\partial x_{1}} \\
-\frac{\partial n}{\partial x_{2}} & \frac{\partial n}{\partial x_{1}} & 0
\end{array}\right)
$$

which is skew symmetric: $Y^{T}=-Y$, and has the further properties that

$$
\begin{aligned}
Y^{2} & =(\nabla n)^{T} \nabla n-(\nabla n)^{2} I, \\
\mathbf{w} Y & =\mathbf{w} \times \nabla n
\end{aligned}
$$

for any row vector $w$, and has the characteristic polynomial $\lambda\left\{\lambda-(\nabla n)^{2}\right\}^{2}$.

$$
\begin{aligned}
\kappa^{2} & =-\mathbf{x}^{\prime} Y^{2} \mathbf{x}^{\prime}, \\
\kappa^{2} \tau & =-\mathbf{x}^{\prime} Y\left(\nabla_{2} n\right) \mathbf{x}^{\prime T} .
\end{aligned}
$$

The knowledge of $\kappa$ and $\tau$ permits us to express the Frenet-Serret formulae for rays

$$
\begin{aligned}
\mathbf{T}^{\prime} & =\quad \kappa n^{-1} \mathbf{N} \\
\mathbf{N}^{\prime} & =-\kappa n^{-1} \mathbf{T} \quad+\tau n^{-1} \mathbf{B} \\
\mathbf{B}^{\prime} & =-\tau n^{-1} \mathbf{N}
\end{aligned}
$$

We now have all the apparatus needed to obtain recursion formulae for the expression of the $m^{\prime}$ th derivative of any vector $\mathrm{y}$ if one knows the coefficients in $\mathrm{y}=a_{0} \mathbf{T}+$ $b_{0} \mathbf{N}+c_{0} \mathbf{B}$ or $\mathbf{y}=d_{0} \mathbf{x}^{\prime}+e_{0} \nabla n+f_{0}\left(\mathbf{x}^{\prime} \times \nabla n\right)$; let us begin with the first form.

If we have

$$
\mathbf{y}^{(m)}=a_{m} \mathbf{T}+b_{m} \mathbf{N}+c_{m} \mathbf{B}
$$

then differentiation and application of Frenet's formulae (15) yield

$$
\mathbf{y}^{(m+1)}=\left\{a_{m}^{\prime}-\kappa n^{-1} b_{m}\right\} \mathbf{T}+\left\{b_{m}^{\prime}+\kappa n^{-1} a_{m}-\tau n^{-1} c_{m}\right\} \mathbf{N}+\left\{c_{m}^{\prime}+\tau n^{-1} b_{m}\right\} \mathbf{B} .
$$

Therefore:

$$
\begin{aligned}
& a_{m+1}=a_{m}^{\prime} \quad-\kappa n^{-1} b_{m} \\
& b_{m+1}=b_{m}^{\prime}+\kappa n^{-1} a_{m} \quad-\tau n^{-1} c_{m}, \\
& c_{m+1}=c_{m}^{\prime} \quad+\tau n^{-1} b_{m}
\end{aligned}
$$


As an example, the first equation of (11) now reads

$$
\text { for } \quad \begin{aligned}
& a_{1}=n^{-1}, \\
& b_{1}=0, \\
& c_{1}=0 .
\end{aligned}
$$

Applying (17) therefore yields

$$
\begin{aligned}
& a_{2}=-n^{\prime} n^{-2}, \\
& \text { for } \quad \mathbf{y}=\mathbf{x}, \quad b_{2}=\kappa n^{-2} \text {, } \\
& c_{2}=0 \text {. }
\end{aligned}
$$

This checks with equation (9) in view of (4) and the second equation of (11).

Similarly, eliminating $\mathbf{x}^{\prime}$ from the first two equations of (11) and solving for $\nabla n$ yields

$$
\text { for } \quad \begin{aligned}
& a_{0}=n n^{\prime}, \\
& b_{0}=n \kappa, \\
& c_{0}=0 .
\end{aligned}
$$

Here again, then, we can apply recursion formulae (17) to give

$$
\text { for } \quad \mathrm{y}=\nabla n, \quad \begin{aligned}
& a_{1}=\left[n n^{\prime}\right]^{\prime}-\kappa^{2}, \\
& b_{1}=[n \kappa]^{\prime}+\kappa n^{\prime}, \\
& c_{1}=\kappa \tau .
\end{aligned}
$$

This formula for $[\nabla n]^{\prime}=\mathbf{x}^{\prime} \nabla_{2} n$ can be given an alternative form, for total derivatives of $n$ and $\kappa$ can always be reduced to expressions involving partial derivatives of $n$ and $\kappa$ with respect to the $x_{i}$ and total derivatives in $\mathbf{x}$. For example, $a_{1}$ is the T component of $[\nabla n]^{\prime}=\mathbf{x}^{\prime} \nabla_{2} n$, hence $a_{1}=\mathbf{x}^{\prime} \nabla_{2} n \cdot \mathbf{T}=n \mathbf{x}^{\prime} \nabla_{2} n \mathbf{x}^{\prime}$.

For the alternative form of $b_{1}$, we need such an expression for $\kappa^{\prime}$, which, in turn, needs one for $n^{\prime \prime}$. From the Lagrange identity and formula (10),

$$
\kappa^{2}=\left|\mathbf{x}^{\prime} \times \nabla n\right|^{2}=n^{-2}(\nabla n)^{2}-n^{\prime 2} .
$$

From this and the alternative expression for $a_{1}$ it is easy to show that

$$
n^{\prime \prime}=\mathbf{x}^{\prime} \nabla_{2} n \mathbf{x}^{\prime \prime}+n^{-3}(\nabla n)^{2}-2 n^{-1} n^{\prime 2} .
$$

Finally, from (23), (12), and a differentiation of (22), we find

$$
\kappa^{\prime}=-2 n^{-1} n^{\prime} \kappa-n^{\prime} \kappa^{-1}\left(\mathbf{x}^{\prime} \nabla_{2} n \mathbf{x}^{\prime T}\right)+n^{-2} \kappa^{-1}\left(\nabla n \nabla_{2} n \mathbf{x}^{\prime T}\right) .
$$

Thus (21) may also be written as follows:

$$
\text { for } \quad \begin{aligned}
& a_{1}=n\left(\mathbf{x}^{\prime} \nabla_{2} n \mathbf{x}^{\prime}\right), \\
& b_{1}=-n n^{\prime} \kappa^{-1}\left(\mathbf{x}^{\prime} \nabla_{2} n \mathbf{x}^{\prime T}\right)+n^{-1} \kappa^{-1}\left(\nabla n \nabla_{2} n \mathbf{x}^{\prime T}\right), \\
& c_{1}=\kappa \tau .
\end{aligned}
$$


This form for $[\nabla n]^{\prime}$ permits us to return to the computation of $\mathbf{x}^{\prime \prime \prime}$ from (19) and (17). Naturally the same result could be obtained by using (23) and (24). In either case a computation yields

$$
a_{3}=-n^{-3}\left\{2 \kappa^{2}-3 n^{\prime 2}+n\left(\mathbf{x}^{\prime} \nabla_{2} n \mathbf{x}^{\prime T}\right)\right\},
$$

for $\quad \mathbf{y}=\mathbf{x}, \quad b_{3}=-n^{-3}\left\{5 n^{\prime} \kappa+\kappa^{-1} n n^{\prime}\left(\mathbf{x}^{\prime} \nabla_{2} n \mathbf{x}^{\prime T}\right)-n^{-1} \kappa^{-1}\left(\nabla n \nabla_{2} n \mathbf{x}^{\prime T}\right)\right\}$,

$$
c_{3}=n^{-3} \kappa \tau \text {. }
$$

Further application of (17) to $\mathbf{y}=\mathbf{x}$ is complicated by the fact that higher order derivatives of $n$ are involved beyond $n^{\prime \prime}$, i.e. beyond those in $\nabla n$ and $\nabla_{2} n$; they are probably best handled by introducing multiple index symbols, such as are used in tensor calculus. At this point the matrix notation stops being convenient. So much for the recursion formulae of type (16). Now let us consider those of type

$$
\mathbf{y}^{(m)}=d_{m} \mathbf{x}^{\prime}+e_{m} \nabla n+f_{m}\left(\mathbf{x}^{\prime} \times \nabla n\right) .
$$

To avoid confusion let us designate the $d_{1}, e_{1}$, and $f_{1}$ when $\mathrm{y}=\nabla n$ by $p_{1}, q_{1}, r_{1}$; if we apply (11) to (21a) we obtain

where

$$
[\nabla n]^{\prime}=p_{1} \mathbf{x}^{\prime}+q_{1} \nabla n+r_{1}\left(\mathbf{x}^{\prime} \times \nabla n\right),
$$

$$
\begin{aligned}
& p_{1}=\kappa^{-2}\left\{(\nabla n)^{2}\left(\mathbf{x}^{\prime} \nabla_{2} n \mathbf{x}^{\prime T}\right)-n^{\prime}\left(\nabla n \nabla_{2} n \mathbf{x}^{\prime T}\right)\right\}, \\
& q_{1}=\kappa^{-2}\left\{-n^{\prime}\left(\mathbf{x}^{\prime} \nabla_{2} n{\mathbf{x}^{\prime}}^{T}\right)+n^{-2}\left(\nabla n \nabla_{2} n{\mathbf{x}^{\prime}}^{T}\right)\right\}, \\
& r_{1}=\tau .
\end{aligned}
$$

Differentiating (26) and using (9) with (27) yields

$$
\begin{aligned}
& d_{m+1}=d_{m}^{\prime}-2 n^{-1} n^{\prime} d_{m}+p_{1} e_{m}+n^{\prime} r_{1} f_{m}, \\
& e_{m+1}=e_{m}^{\prime}+n^{-3} d_{m} \quad+q_{1} e_{m}-n^{-2} r_{1} f_{m} \\
& f_{m+1}=f_{m}^{\prime} \quad+r_{1} e_{m}+\left\{q_{1}-2 n^{-1} n^{\prime}\right\} f_{m} .
\end{aligned}
$$

As examples, if $\mathbf{y}=\mathbf{x}$ in (26) and $m=1$, then obviously $d_{1}=1, e_{1}=0, f_{1}=0$; hence, from (28), $d_{2}=-2 n^{-1} n^{\prime}, e_{2}=n^{-3}, f_{2}=0$, which is the same as (9). Another application of (28) with $m=2$ yields

$$
\begin{aligned}
& d_{3}=-2\left(n^{-1} n^{\prime}\right)^{\prime}+4 n^{-2} n^{\prime 2}+n^{-3} p_{1}, \\
& \text { for } \quad \mathbf{y}=\mathbf{x}, \quad e_{3}=\left(n^{-3}\right)^{\prime}-2 n^{-4} n^{\prime} \quad+n^{-3} q_{1} \text {, } \\
& f_{3}=n^{-3} r_{1} \text {. }
\end{aligned}
$$

Again, if $\mathbf{y}=\nabla n$, then $d_{0}=0, e_{0}=1, f_{0}=0$, hence $d_{1}=p_{1}, e_{1}=q_{1}, f_{1}=r_{1}$ as in (27).

To obtain power series expansions of the rays

$$
\mathbf{x}=\mathbf{x}_{0}+\mathbf{x}_{0}^{\prime} u+\frac{1}{2} \mathbf{x}_{0}^{\prime \prime} u^{2}+\cdots+\frac{1}{m !} \mathbf{x}_{0}^{(m)} u^{m}+\cdots,
$$

one can either apply (16) to $\mathbf{y}=\mathbf{x}$ at $u=0$ (the first four terms come from (18), (19), and (25) if one desires to use $\mathbf{T}, \mathbf{N}$, and $\mathbf{B}$ as coordinate vectors), or one can apply (26) and use the recursion formulae (28), as just indicated. 


\section{REFERENCES}

1. M. Herzberger, Strahlenoptik, Springer, Berlin, 1931.

2. M. Herzberger, Zur Optik inhomogener. Mittel, Zeitschrift für Instrumentenkunde, 53, 436-443 (1933).

3. J. I. Singe, Geometrical optics, Cambridge Tracts No. 37, 1937.

4. R. K. Luneberg, Mathematical theory of optics, Brown University mimeographed lecture notes, Providence, R. I., 1944.

5. P. Franklin, .Ycthods of advanced calculus, McGraw-Hill Book Co., New York, 1944.

6. S. Gorn, Rays in isotropic, non-homogeneous media, Air Force Technical Report No. 6262 (1951).

\section{ON SUPERSONIC FLOW PAST AN OSCILLATING WEDGE*}

\section{By MILTON D. VAN DYKE (Ames Aeronautical Laboratory, Moffett Field, Calif.)}

1. Introduction. In order to study the limitations of the linearized theory of oscillating airfoils, Carrier ${ }^{1}$ has analyzed supersonic flow past a thick wedge which oscillates slightly about its apex. Considerably greater insight into the nonlinear effects of thickness is gained by generalizing the analysis to include other locations of the pivot.

2. Analysis. Consider a wedge fixed in a supersonic stream at a Mach number above that at which the shock wave detaches from the apex. If now the wedge executes slight oscillations, the shock will remain attached, and the flow field can be found by superposing a linearized acoustic field upon the nonlinear steady flow. For harmonic oscillations of frequency $a_{2} c$, Carrier has shown ${ }^{1,2}$ that the velocity components and pressure downstream of the shock are given by

$$
\begin{aligned}
& u=U_{2}+a_{2}\left(\varphi_{x}+E_{y}\right), \quad v=a_{2}\left(\varphi_{y}-E_{x}\right), \\
& p=p_{2}\left[1-\gamma\left(\varphi_{\imath}+M \varphi_{x}\right)\right],
\end{aligned}
$$

where

$$
\begin{aligned}
& \varphi=e^{i c\left(t-M x / \beta^{2}\right)} \sum^{\infty}\left(a_{\nu} \cosh \nu \theta+b_{\nu} \sinh \nu \theta\right) J_{\nu}(k r), \\
& E=e^{i r\left(t-x / M-\lambda_{y} / M \beta^{2}\right)} \sum^{\infty} c_{\nu} J_{\nu}(k \xi y),
\end{aligned}
$$

and the deflection of the shock wave from its steady position is

$$
\psi=\left(1-\rho_{1} / \rho_{2}\right)^{-1} e^{i c\left(t-\lambda . M_{v} / \beta^{2}\right)} \sum^{\infty} d_{\nu} J_{v}(k \xi y) .
$$

Here, $U_{2}, a_{2}, M, p_{2}$ and $\rho_{2}$ are the flow speed, sound speed, Mach number, pressure and density downstream of the shock in steady flow (and $\rho_{1}$ the density upstream), $\gamma$ is the adiabatic exponent, $t$ the time multiplied by $a_{2}, \beta^{2}=M^{2}-1, k=c / \beta^{2}, \tanh \theta=$ $\beta y / x, r^{2}=x^{2}-\beta^{2} y^{2}$ and $\xi^{2}=1+\lambda^{2}-M^{2}$. The coordinate system and $\lambda$ are defined in Fig. 1.

*Received Feb. 18, 1953.

${ }^{1} \mathrm{G}$. F. Carrier, The oscillating wedge in a supersonic stream, J. Aer. Sci., 16, 150-152 (1949).

${ }^{2} \mathrm{G}$. F. Carrier, On the stability of the supersonic flows past a wedge, Q. Appl. Math. 6, 356-378 (1949). 